\title{
The use of different pathology classification systems in preoperative imaging of Mirizzi syndrome
}

Ya Feng Ji, Yu Gao, Min Xie

Department of Radiology, the Second People's Hospital of Wuxi, Wuxi, Jiangsu, China

Submitted: 20 February 2017

Accepted: 11 June 2017

Arch Med Sci 2019; 15 (5): 1288-1293

DOI: https://doi.org/10.5114/aoms.2019.87131

Copyright @ 2019 Termedia \& Banach

\section{Abstract}

Introduction: The aim was to evaluate the diagnostic value of the Nakagawa and Csendes pathology classification systems in preoperative imaging of Mirizzi syndrome. Mirizzi syndrome is a type of biliary system obstruction caused by stones impacted in a gallbladder neck or cystic duct situated parallel to the common bile duct, causing extrinsic common bile duct stenosis or obstruction, which can lead to recurrent obstructive jaundice, bile duct erosion, and cholangitis. Therefore, the preoperative identification and classification of Mirizzi syndrome is vital for a good surgical result. We explored the applicability of two pathological classification systems to diagnostic imaging.

Material and methods: We performed a retrospective analysis of the clinical, computed tomography, and magnetic resonance imaging data of 76 cases of pathologically confirmed Mirizzi syndrome, comparing the applicability of the Csendes and Nakagawa pathology classification systems to preoperative imaging.

Results: The Nagakawa pathology classification system had higher sensitivity, specificity, accuracy, positive predictive value, and positive likelihood ratio, along with lower rates of both missed diagnosis and misdiagnosis. Its positive predictive value and positive likelihood ratios were significantly superior. Adapting the Nagakawa pathological classification system to preoperative imaging produced more consistent results than the Csendes system. Conclusions: Compared with the Csendes pathology classification system, the Nagakawa classification is more adaptable to preoperative imaging and treatment planning.

Key words: Mirizzi syndrome/CL, computed tomography, magnetic resonance imaging.

\section{Introduction}

Mirizzi syndrome is a type of biliary system obstruction caused by stones impacted in a gallbladder neck or cystic duct situated parallel to the common bile duct, causing extrinsic common bile duct stenosis or obstruction, which can lead to recurrent obstructive jaundice, bile duct erosion, and cholangitis [1-3]. The preoperative diagnosis of Mirizzi syndrome is difficult, and if it is not diagnosed correctly before surgery, there is an elevated risk of intraoperative bile duct injury $[4,5]$. Therefore, the preoperative identification and classification of Mirizzi syndrome is vital to a good surgical result [6-8]. Mirizzi syndrome classification is a pathological classification; there is no standard imaging classification.

\author{
Corresponding author: \\ Yu Gao MD \\ Department \\ of Radiology \\ Wuxi No. 2 People's \\ Hospital \\ 68 Zhongshan Road \\ Wuxi, Jiangsu 214002 \\ China \\ E-mail: 2669515289@qq.com
}


In this study, we explored the applicability of two pathological classification systems to diagnostic imaging.

\section{Material and methods}

We reviewed the cases of 76 patients clinically suspected of having Mirizzi syndrome, who were treated in our hospital over almost 5 years (20102015), and who had complete clinical and imaging data.

The patients included 31 males and 45 females aged $37-80$ years (mean: 56.5 ). All patients were the same when using different imaging classification. All patients were selected according to the following criteria. (1) Complete clinical and pathological imaging information available. (2) Patients with Mirizzi syndrome confirmed by pathology. (3) Patients with serious bile duct diseases, such as cholangiocarcinoma, were excluded from the study. Of these, 58 patients had had unexplained right upper quadrant pain ranging from 1 week to 2 years. On physical examination, 54 patients had right upper quadrant tenderness and 11 patients had rebound tenderness. Laboratory studies revealed 45 patients with elevated serum $\gamma$ glutamyl transferase (GGT), 1.2$3.41 \mu \mathrm{kat} / \mathrm{l}$ (normal: 0.13-0.97 $\mu \mathrm{kat} / \mathrm{l}), 34$ patients with hyperbilirubinemia, 25-38 $\mu \mathrm{mol} / \mathrm{l}$ (normal: 3.4-20 $\mu \mathrm{mol} / \mathrm{l}), 33$ patients with elevated serum alanine aminotransferase (ALT), 1.12-2.42 $\mu \mathrm{kat} / \mathrm{l}$ (normal: 0.08-0.83 $\mu \mathrm{kat} / \mathrm{l}$ ), 12 patients with elevated alkaline phosphatase, 0.9-2.14 $\mu \mathrm{kat} / \mathrm{l}$ (normal: 0.38-0.62 $\mu \mathrm{kat} / \mathrm{l}), 11$ patients with elevated lactate dehydrogenase (LDH), 4.54-6.26 $\mu \mathrm{kat} / \mathrm{l}$ (normal: 1.74-4.09 $\mu \mathrm{kat} / \mathrm{l})$, and 9 patients with elevated serum aspartate aminotransferase, 1.09-1.97 $\mu \mathrm{kat} / \mathrm{l}$ (normal: 0.08-0.83 $\mu \mathrm{kat} / \mathrm{l}$ ).

\section{Imaging}

A 64-slice spiral CT scanner (Aquilion, Toshiba Medical Systems, Otawara, Japan) was used in this study. The research was performed according to the World Medical Association Declaration of Helsinki. The patients were scanned from the diaphragm to the pubic symphysis. After a noncontrast acquisition, dual-phase enhanced scanning was performed following intravenous injection of 1.5-2.0 $\mathrm{ml} / \mathrm{kg}$ of iopromide (Ultravist, Schering, Berlin, Germany) with a high-pressure injector at a rate of $2.0-3.0 \mathrm{ml} / \mathrm{s}$ (arterial phase delay $20-25 \mathrm{~s}$, portal venous phase delay $60-70 \mathrm{~s}$ ) using the following settings: $100-120 \mathrm{kV}, 150-180 \mathrm{~mA}, 10 \mathrm{~mm}$ section thickness, $10 \mathrm{~mm}$ of the layer spacing, pitch $=1.0$. After scanning, data were sent to the workstation for three-dimensional reconstruction.

MRI (1.5 T HD Twin Speed General Electric, Milwaukee WI, USA) with an eight- channel phased-array body coil was used to acquire images from the liver dome through the pubic symphysis. First, respiratory-gated and fast spin-echo (FSE) axial T2-weighted images (T2WI) were acquired with a $228 \times 224$ matrix. Subsequently, MR cholangiopancreatography (MRCP) was performed with $6 \mathrm{~mm}$ section thickness and $10 \mathrm{~mm}$ layer spacing, followed by breath-hold three-dimensional fast spoiled gradient echo T1WI axial images. In the enhanced scanning, a three-dimensional ultrafast multi-phase enhanced scanning sequence was used. The contrast agent was Gd-DTPA.

$3 \mathrm{D}$ reconstruction were analyzed by two experienced chief radiologists who were blinded to the pathology results. They solved problems when they were not in agreement. They were good at abdominal imaging diagnosis.

\section{Statistical analysis}

Both the Csendes and Nakagawa pathological classification systems were applied to imaging findings, and compared with the pathologic results. We calculated the sensitivity, specificity, accuracy, the rate of missed diagnosis, misdiagnosis rate, positive predictive value, negative predictive value, positive likelihood ratio, and negative likelihood ratio. We compared the correlation ( $\kappa$ consistency test and 95\% Cl) between each image classification and pathological classification.

\section{Results}

\section{Pathological results}

All 76 patients underwent open cholecystectomy with choledochobiliary fistula repair, choledochoplasty, or bilioenteric anastomosis performed based on the imaging findings and the surgeons' experience. Their pathologies were all chronic cholecystitis with cholelithiasis. Under the microscope, gallbladder epithelial atrophy was observed with wall fibrosis and lymphocyte infiltration.

\section{Imaging results}

Using the 1989 Csendes classification, computed tomography (CT) and magnetic resonance imaging (MRI) classified 41 patients as type I, 5 patients as type II, 8 patients as type III, and 5 patients as type IV. Seventeen patients were diagnosed with disease other than Mirizzi syndrome. According to the 1997 Nagakawa classification, CT and MRI classified 41 patients as type I, 18 patients as type II, 10 as type III, 4 patients as type IV, and 3 patients without Mirizzi syndrome (Figure 1).

\section{Pathological classification}

According to the 1989 Csendes classification, CT and MRI classified 36 patients as type I, 16 pa- 

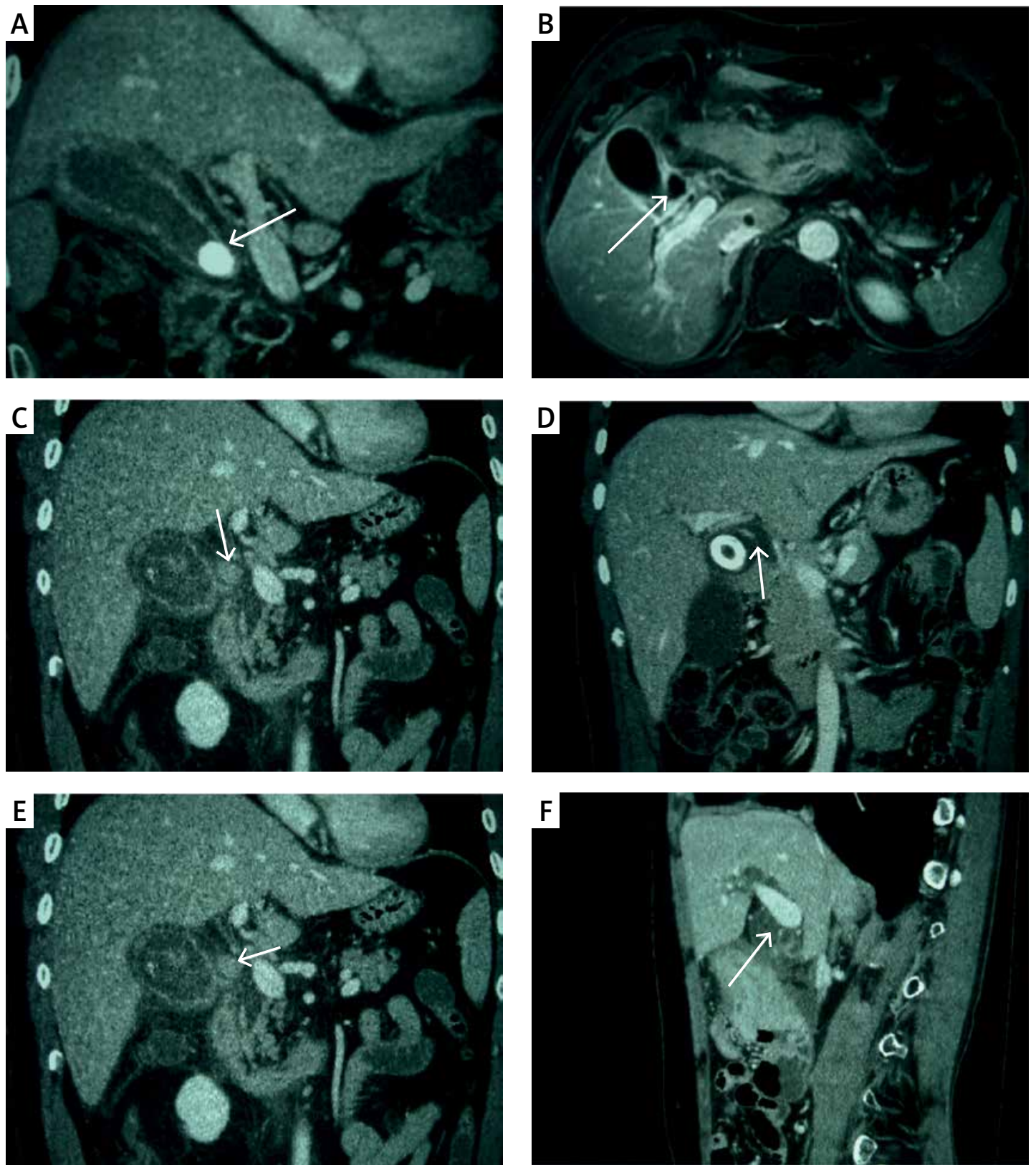

Figure 1. A - CT coronal scan in portal venous phase shows gallbladder neck incarcerated, oppression of common hepatic duct (white arrow). B - MRI contrast-enhanced T1WI shows gallbladder neck incarcerated (white arrow), with the level of hepatic duct stenosis, belonging to Nagakawa type I. C - CT coronal scan in portal venous phase shows gallbladder neck incarcerated (white arrow), with the level of hepatic duct stenosis, belonging to Nagakawa type I. D - CT coronal scan in portal venous phase shows gallbladder and common hepatic duct fistula (white arrow), with $2 / 3$ of the fistula over the circumference of the biliary tract, belonging to Nagakawa type II. E - CT coronal scan in portal venous phase shows stone located in the confluence of cystic duct and common bile duct (white arrow), belonging to Nagakawa type III. F - CT sagittal scan in portal venous phase shows no stone in cystic duct and gallbladder neck (white arrow), with inflammatory stricture of common bile duct, belonging to Nagakawa type IV

tients as type II, 4 patients as type III, 1 patient as type IV, and 19 as another diagnosis.

According to the 1997 Nagakawa classification, CT and MRI classified 36 patients as type I, 21 patients as type II, 12 patients as type III, 5 patients as type IV, and 2 patients as another diagnosis.

\section{Statistical evaluation}

The accuracy analysis of the two pathology classification systems was evaluated. They only have differences in positive predictive value and positive likelihood ratio (Table I), suggesting that both pathology classification systems have simi- 
lar results for imaging diagnosis. The correlation between imaging and pathological classifications was evaluated.

According to the Csendes classification as ascertained by imaging, 41 cases were type I, 5 were type II, 8 were type III, 5 were type IV, and 17 had another diagnosis.

According to findings in pathology, there were 36 type I patients, 16 type II, 4 type III, 1 type IV, and 19 had another diagnosis. The $\kappa$ consistency examination on image classification and pathological classification was done and $\kappa=0.57$ (Table II), suggesting that the correlation was acceptable. According to the Nagakawa classification as ascertained by imaging, 41 cases were type I, 18 were type II, 10 were type III, 4 were type IV, and 3 had another diagnosis. According to the pathological classification, there were 36 type I patients, 21 type II, 12 type III, 5 type IV, and 2 had another diagnosis. The $\kappa$ consistency test on image classification and pathological classification showed $\kappa=0.68$ (Table III), suggesting a good correlation. Ridit analysis (95\% confidence interval) shows that (sendes pathology classification and Nagakawa pathology classification, Csendes pathology classification and
Csendes imaging classification, and Nagakawa pathology classification and Nagakawa imaging classification have no significant statistical difference $(p>0.05)$ (Tables I, IV).

\section{Discussion}

Mirizzi syndrome is extraneous compression of the common bile duct by a stone or stones in the gallbladder neck or in an anatomically variant cystic duct situated parallel to the common duct. The first reported case dates back to the early $20^{\text {th }}$ century [9] and was named after the Argentinean surgeon Mirizzi in 1948.

Preoperative diagnosis of Mirizzi syndrome is difficult, and intraoperative bile duct injury can easily occur in unsuspected cases, which can cause serious complications. Preoperative imaging can help the surgeon to avoid confusing the common bile duct with the parallel cystic duct.

The surgical treatment of Mirizzi syndrome is related to its type [10-12]. In 1989, Csendes et al. [13] divided Mirizzi syndrome into four types according to the degree of common bile duct damage, in which type I featured extrinsic common

Table I. The accuracy analysis of the two pathology classification systems in these patients

\begin{tabular}{|lccc|}
\hline Parameter & $\begin{array}{c}\text { Csendes classification with } \\
95 \% \mathrm{Cl}\end{array}$ & $\begin{array}{c}\text { Nagakawa classification with } \\
95 \% \mathrm{Cl}\end{array}$ & $\boldsymbol{P}$-value \\
\hline Sensitivity & $0.97(0.9478-0.9922)$ & $0.99(0.9678-1.0000)$ & $>05$ \\
\hline Specificity & $0.79(0.6984-0.8816)$ & $1.00(1.0000-1.0000)$ & $>.05$ \\
\hline Accuracy & $0.92(0.8590-0.9810)$ & $0.99(0.9678-1.0000)$ & $>05$ \\
\hline Missed diagnosis rate & $0.03(0.0000-0.0684)$ & $0.01(0.0000-0.0324)$ & $>05$ \\
\hline Misdiagnosis rate & $0.21(0.1184-0.3016)$ & $0.00(0.0000-0.0000)$ & $>0.05$ \\
\hline Positive predictive value & $0.93(0.8726-0.9874)$ & $1.00(1.0000-1.0000)$ & $>0.05$ \\
\hline Negative predictive value & $0.88(0.8069-0.9531)$ & $0.67(0.5643-0.7757)$ & $>0.05$ \\
\hline Positive likelihood ratio & $4.58(3.1422-6.7899)$ & $\infty(\infty-\infty)$ & $>0.05$ \\
\hline Negative likelihood ratio & $0.04(0.0095-0.1516)$ & $0.01(0.0014-0.0703)$ & $>0.05$ \\
\hline Total 95\% Cl & 0.5000 & $0.3756-0.5080$ & $>$
\end{tabular}

Table II. Consistency between image classification and pathological classification (Csendes classification)

\begin{tabular}{|c|c|c|c|c|c|c|}
\hline $\begin{array}{l}\text { Pathology } \\
\text { image }\end{array}$ & I & II & III & IV & None & Total \\
\hline I & 31 & 10 & 0 & 0 & 0 & 41 \\
\hline II & 1 & 4 & 0 & 0 & 0 & 5 \\
\hline III & 3 & 0 & 3 & 0 & 2 & 8 \\
\hline IV & 0 & 1 & 1 & 1 & 2 & 5 \\
\hline None & 1 & 1 & 0 & 0 & 15 & 17 \\
\hline Total & 36 & 16 & 4 & 1 & 19 & 76 \\
\hline
\end{tabular}


Table III. Consistency between image classification and pathological classification (Nagakawa classification)

\begin{tabular}{|lcccccc|}
\hline $\begin{array}{l}\text { Pathology } \\
\text { image }\end{array}$ & I & II & III & IV & None & Total \\
\hline I & 31 & 7 & 3 & 0 & 0 & 41 \\
\hline II & 3 & 14 & 0 & 1 & 0 & 18 \\
\hline III & 1 & 0 & 9 & 0 & 0 & 10 \\
\hline IV & 0 & 0 & 0 & 4 & 0 & 4 \\
\hline None & 1 & 0 & 0 & 0 & 2 & 3 \\
\hline Total & 36 & 21 & 12 & 5 & 2 & 76 \\
\hline
\end{tabular}

Table IV. Ridit analysis $(95 \% \mathrm{Cl})$

\begin{tabular}{|lcc|}
\hline Csendes pathology classification & Csendes imaging classification & $P$-value \\
\hline 0.5000 & $0.4111-0.5435$ & $>0.05$ \\
\hline Nagakawa pathology classification & Nagakawa imaging classification & $P$-value \\
\hline 0.5000 & $0.4039-0.5363$ & $>0.05$ \\
\hline Csendes pathology classification & Nagakawa pathology classification & $P$-value \\
\hline 0.5000 & $0.3756-0.5080$ & $>0.05$ \\
\hline
\end{tabular}

bile duct compression by stones in the cystic duct or gallbladder neck; type II was further complicated by a cholecystobiliary fistula involving less than $1 / 3$ of the common bile duct circumference; type III featured a fistula involving up to $2 / 3$ of-the common bile duct circumference; and type IV featured complete destruction of the common bile duct. In 1997, Nagakawa et al. [14] proposed a new classification method, in which type I again featured extrinsic common bile duct compression by stones in the cystic duct or gallbladder neck; type II featured a cholecystobiliary fistula; type III had stones located at the confluence of the cystic duct and common hepatic duct; and type IV had no stones in the cystic duct or gallbladder neck, but featured inflammation around a common hepatic duct stricture induced by compression by cholecystitis with or without stones in the gallbladder. In the Nagakawa classification, Csendes types II-IV correspond to Nakagawa type II, and types III and IV are exclusive to the Nakagawa classification.

These classifications of Mirizzi syndrome are pathological, and the preoperative classification of Mirizzi syndrome can only rely on diagnostic imaging. As ultrasound often cannot visualize the common bile duct, Mirizzi syndrome assessment is mainly dependent on CT and MRI examinations [15]. We believe it is for the following reasons: the Csendes classification focuses on the measurement of fistula size, but it is difficult to estimate the amount of common bile duct circumferential involvement on CT or MRCP. The Nagakawa clas- sification does not require quantification of bile duct fistula involvement, but only requires ruling in or out a cholecystobiliary fistula, assessing biliary stones, and detecting common bile duct in flammatory strictures, which are well within the capabilities of CT and MRI/MRCP. The Nagakawa classification was higher in sensitivity, specificity, accuracy, positive predictive value, positive likelihood ratio, Youden index, with lower rates of both missed diagnosis and misdiagnosis. There was a significant difference in positive predictive value and positive likelihood ratio in favor of the Nagakawa system. The $\kappa$ consistency test showed that the Nagakawa classification had a better correlation between imaging classification and pathological classification though Ridit analysis $(95 \% \mathrm{Cl})$ shows no statistically significant difference.

In conclusion, in this study, imaging was used as an approach to simulated preoperative pathological classification, based on the Csendes and Nagakawa classification systems. The results of this study showed that, compared with the Csendes classification system, the Nagakawa classification system was superior to the Csendes system in many statistical indexes for diagnostic accuracy, especially as the consistency between Csendes imaging classification and pathological classification was low. Compared with the Csendes classification, the Nagakawa classification is more suitable for image-based preoperative planning.

\section{Conflict of interest}

The authors declare no conflict of interest. 


\section{References}

1. Kujawski K, Stasiak M, Rysz J. Qualification for endoscopic retrograde cholangiopancreatography in the diagnosis and treatment of extrahepatic cholestasis caused by choledocholithiasis. Arch Med Sci 2015; 11: 1213-6.

2. Johnson AG, Hosking SW. Appraisal of the management of bile duct stones. Br J Surg 1987; 74: 555-60.

3. Halldestam I, Enell EL, Kullman E, Borch K. Development of symptoms and complications in individuals with asymptomatic gallstones. Br J Surg 2004; 91: 734-8.

4. Kwon AH, Inui H. Preoperative diagnosis and efficacy of laparoscopic procedures in the treatment of Mirizzi syndrome. J Am Coll Surg 2007; 204: 409-15.

5. Waisberg J, Corona A, de Abreu IW, Farah JF, Lupinacci RA, Goffi FS. Benign obstruction of the common hepatic duct (Mirizzi syndrome): diagnosis and operative management. Arq Gastroenterol 2005; 42: 13-8.

6. Williams EJ, Green J, Beckingham I, Parks R, Martin D, Lombard M. Guidelines on the management of common bile duct stones. Gut 2008; 57: 1004-21.

7. Frossard JL, Morel PM. Detection and management of bile duct stones. Gastrointest Endosc 2010; 72: 808-16.

8. Maple JT, Ben-Menachem T, Anderson MA, et al. The role of endoscopy in the evaluatiocholithiasis. Gastrointest Endosc 2010; 71: 1-9.

9. Beltran MA. Mirizzi syndrome: history, current knowledge and proposal of a simplified classification. World J Gastroenterol 2012; 18: 4639-50.

10. Wijesuriya SR, Delriviere L, Mitchell A. Gall bladder cancer and Mirizzi syndrome: alternative explanation to the common belief. ANZ J Surg 2010; 80: 116-7.

11. Lampropoulos P, Paschalidis N, Marinis A, Rizos S. Mirizzi syndrome type $V a$ : a rare coexistence of double cholecysto-biliary and cholecysto-enteric fistulae. World J Radiol 2010; 2: 410-3.

12. Presta L, Ragozzino A, Perrotti P, et al. Detection of Mirizzi syndrome with magnetic resonance cholangiopancreatography: laparoscopic or pen approach. Surg Endosc 2002; 16: 1494-5.

13. Csendes A, Dlaz JC, Burdihs P, Maluenda F, Nava O. Mirizzi syndrome and cholecystobiliary fistula: a unifying classification. Br J Surg 1989; 76: 1139-43.

14. Nagakawa T, Ohta T, Kayahara M. A new classification of Mirizzi syndrome from diagnostic and therapeutic viewpoints. Hepatogastroenterology 1997; 44: 63-7.

15. Zhong H, Gong JP. Mirizzi syndrome: experience in diagnosis and treatment of 25 cases. Am Surg 2012; 78: 61-5. 